\title{
BMJ Open Risk factors for new chronic opioid use after hip fracture surgery: a Danish nationwide cohort study from 2005 to 2016 using the Danish multidisciplinary hip fracture registry
}

To cite: Edwards NMcK, Varnum C, Overgaard S, et al. Risk factors for new chronic opioid use after hip fracture surgery: a Danish nationwide cohort study from 2005 to 2016 using the Danish multidisciplinary hip fracture registry. BMJ Open 2021;11:e039238. doi:10.1136/ bmjopen-2020-039238

- Prepublication history and additional material are published online only. To view please, visit the journal online (http://dx.doi. org/10.1136/bmjopen-2020039238).

Received 08 April 2020 Revised 01 February 2021 Accepted 12 February 2021

Check for updates

(C) Author(s) (or their employer(s)) 2021. Re-use permitted under CC BY-NC. No commercial re-use. See rights and permissions. Published by BMJ.

For numbered affiliations see end of article.

Correspondence to Nina McKinnon Edwards; nme@clin.au.dk

\section{ABSTRACT}

Objective To examine the risk factors for new chronic opioid use in elderly patients who underwent hip fracture surgery.

Design Prospective population-based cohort study. Setting and participants Using Danish nationwide health registries, we identified all opioid non-user patients aged $\geq 65$ years who had undergone hip fracture surgery from 2005 to 2016 and were alive within the first year following surgery.

Main outcome measures New chronic opioid use defined by the dispensing of at least two prescription opioids within two of the last three quarters during the first year following surgery.

Results We identified 37202 opioid non-user patients who underwent hip fracture surgery. Of these, 5497 (15\%) developed new chronic opioid user within 1 year of surgery. Risk factors for new chronic opioid use were Body Mass Index (BMI) of <18.5 (adjusted OR (a0R) 1.22, 95\% Cl 1.09 to 1.36), BMl of 25.0-29.9 (aOR 1.12, 95\% Cl 1.04 to 1.21) and $\mathrm{BMl}$ of $\geq 30$ (aOR $1.57,95 \% \mathrm{Cl} 1.40$ to 1.76 ) with $\mathrm{BMI}$ 18.6-24.9 as reference, a pertrochanteric/subtrochanteric fracture (aOR 1.27, $95 \% \mathrm{Cl} 1.20$ to 1.34) with femoral neck fracture as reference, preoperative use (vs no-use) of nonsteroidal anti-inflammatory drug (aOR 1.68, 95\% Cl 1.55 to 1.83), selective serotonin reuptake inhibitor (aOR 1.42, 95\% $\mathrm{Cl} 1.32$ to 1.53), antidepressants (aOR 1.36, 95\% Cl 1.24 to 1.49 ), antipsychotics (aOR 1.21, $95 \% \mathrm{Cl} 1.07$ to 1.35), corticosteroids (aOR 1.54, 95\% Cl 1.35 to 1.76), statins (aOR 1.09, 95\% Cl 1.02 to 1.18), antibiotics (aOR 1.32, $95 \% \mathrm{Cl} 1.22$ to 1.42), antiosteoporosis drugs (a0R 1.33, $95 \% \mathrm{Cl} 1.19$ to 1.49 ) and anticoagulatives (aOR 1.24, 95\% $\mathrm{Cl} 1.17$ to 1.32). Presence of cardiovascular comorbidities, diabetes, gastrointestinal diseases, dementia, chronic obstructive pulmonary disease or renal diseases was further identified as a risk factor.

Conclusion In this large nationwide cohort study, we identified several risk factors associated with new chronic opioid use after hip fracture surgery among patients who were alive within the first year following surgery. Although not all factors are modifiable preoperative, this will allow clinicians to appropriately counsel patients preoperatively and tailor postoperative treatment.

\section{Strengths and limitations of this study}

- This study is a prospective population-based cohort study with complete follow-up based on Danish nationwide health registries.

- The study includes comprehensive high-quality data on medication use and comorbidities before surgery, and detailed clinical and opioid data from registries rather than patient-reported data.

- The definition of new chronic opioid use is inspired by the guidelines from the International Association for the Study of Pain.

- Neither data on clinical indications for opioid prescriptions and patient compliance with opioid treatment nor data on reoperations during follow-up were available.

- In our primary analysis, we excluded all deceased patients within the first year following surgery. This might have compromised the external validity of our study and potentially introduced immortal time. We used a number of sensitivity analyses, including landmark analysis to test the robustness of our estimates. Thus, we can only conclude that the identified risk factors for new chronic opioid use apply for patients with hip fracture who survived 1 year after surgery.

\section{INTRODUCTION}

The prevalence of hip fractures is estimated to reach 6.3 million people worldwide by 2050. ${ }^{1}$ Patients with hip fracture often suffer from comorbidities and polypharmacy, which have been associated with an increased risk of complications and increased mortality. In addition, postsurgical pain can delay mobilisation and rehabilitation. ${ }^{2}$ These factors make treatment and rehabilitation for patients who underwent hip fracture surgery challenging.

Opioids are commonly accepted as standard clinical practice for pain treatment in patients who had hip fracture surgery. However, initial 
prescription opioid treatment for acute postsurgical pain still merits caution as it can result in chronic opioid use. ${ }^{3}$ Previous studies have shown that a high percentage of patients with hip fracture who did not use opioids before their hip fracture were still using opioids several months after surgery. ${ }^{45}$ This is concerning as chronic prescription opioid use can have a negative impact on quality of life, ${ }^{6}$ has been associated with increased risk of sustaining new fractures ${ }^{7}$ and other adverse events, including general medical complications. ${ }^{3}$

The risk of chronic opioid use can be influenced by patient-related, surgical and healthcare-related factors, some of which are modifiable (weight, medication and surgery type), whereas others are non-modifiable (age and gender). Only few studies have investigated risk factors for chronic opioid use in orthopaedic patients. Moreover, these studies are limited by small sample sizes, study populations that differ from the hip fracture population, varying definitions of opioid use, different follow-up durations and lack of adjustment for potential confounders. ${ }^{89}$ No previous studies have investigated risk factors for chronic opioid use after hip fracture surgery. Thus, there is a need for more knowledge on risk factors for new chronic opioid use in patients with hip fracture, in particular, risk factors that are modifiable during preoperative, perioperative and postoperative period.

The aim of this study was to examine patient-related and surgery-related risk factors associated with new chronic opioid use in elderly patients who underwent hip fracture surgery using nationwide health registries.

\section{PATIENTS AND METHODS}

\section{Study design and setting}

We conducted this population-based cohort study in Denmark using prospectively collected administrative health data from health registries, which cover all contacts to the health sector. ${ }^{10}$ The Danish National Health Services provide tax-supported primary and secondary healthcare for the entire population, ${ }^{10}$ ensuring that all eligible patients with hip fracture undergo surgery at a public hospital. All Danish citizens can be identified using a 10-digit personal identification number, which goes through all Danish health registries allowing for unambiguous individually record linkage of data. ${ }^{11}$

\section{Data sources}

The Danish Civil Registration System was initiated in 1968. Daily updated information on migration and vital status allows for virtually complete long-term follow-up on emigration and death. ${ }^{11}$

The Danish Multidisciplinary Hip Fracture Registry (DMHFR) was initiated in 2003 and contains nationwide population-based data about all patients undergoing primary hip fracture surgery. ${ }^{12}$ The positive predictive value of the hip fracture diagnosis is between $90 \%$ and $98 \%$ depending on fracture type. ${ }^{13}$
The Danish National Health Service Prescription Database (DNHSPD) has kept information on all prescriptions for reimbursed drugs dispensed by community pharmacies in Denmark since 2004 according to Anatomical Therapeutic Chemical (ATC) classification system (ATC codes). Data from the DNHSPD can account for patient's medication. ${ }^{14}$

The Danish National Patient Registry (DNPR) is an administrative registry established in 1977 covering all somatic contacts in all Danish hospitals. ${ }^{15}$ Information reported to the DNPR includes administrative data, diagnoses, treatments and examinations. Primary and secondary diagnoses are reported to the DNPR according to the International Disease Classification, 10th revision, since $1995 .^{15}$ The positive predictive value of the diagnoses included in the medical comorbidities is more than $90 \% .^{16}$

\section{Study population}

We used the DMHFR to identify all patients aged 65 years or older who were treated for a fracture of the femoral neck, pertrochanteric or subtrochanteric fracture with osteosynthesis, or total/partial hip replacement surgery between 1 January 2005 and 31 December 2015 (online supplemental appendix 1). Patients were indexed on their surgery date and followed up for 12 months (to 31 December 2016). Patients who had dispensed an opioid in the 6 months prior to index were excluded to ensure they were opioid non-users at the index date. Patients who died from any cause within 12 months following their index date were also excluded (figure 1).

\section{Outcome}

We characterised patients with new chronic opioid use as having dispensed an opioid prescription in at least two of the three last quarters (3-month periods per quarter) within the first year following surgery among previously opioid non-user patients (figure 2). ${ }^{8} 17$ Prescription opioids dispensed by community pharmacies 7 days prior to index and within the first quarter after index were not included in the outcome definition because they are likely to have been associated with the initial opioid treatment to manage acute postoperative pain. ${ }^{4}$ Thus, we were only interested in subsequent opioid prescriptions beyond the early perioperative period (quarters 2-4). Our definition of chronic opioid use after surgery was aligned with the definition from the International Association for the Study of Pain, which defines chronic postsurgical pain as pain that develops after a surgical procedure and persists for at least 3 months after surgery. ${ }^{18}$

The following prescription opioids were included in the analysis: morphine, hydromorphine, nicomorphine, oxycodone, oxycodone combined with naloxone, pethidine, fentanyl, ketobemidone, methadone, codeine, tramadol, tapentadol and buprenorphine.

\section{Risk factors}

Based on previous literature and clinical experience, the below-mentioned patient-related and surgery-related 

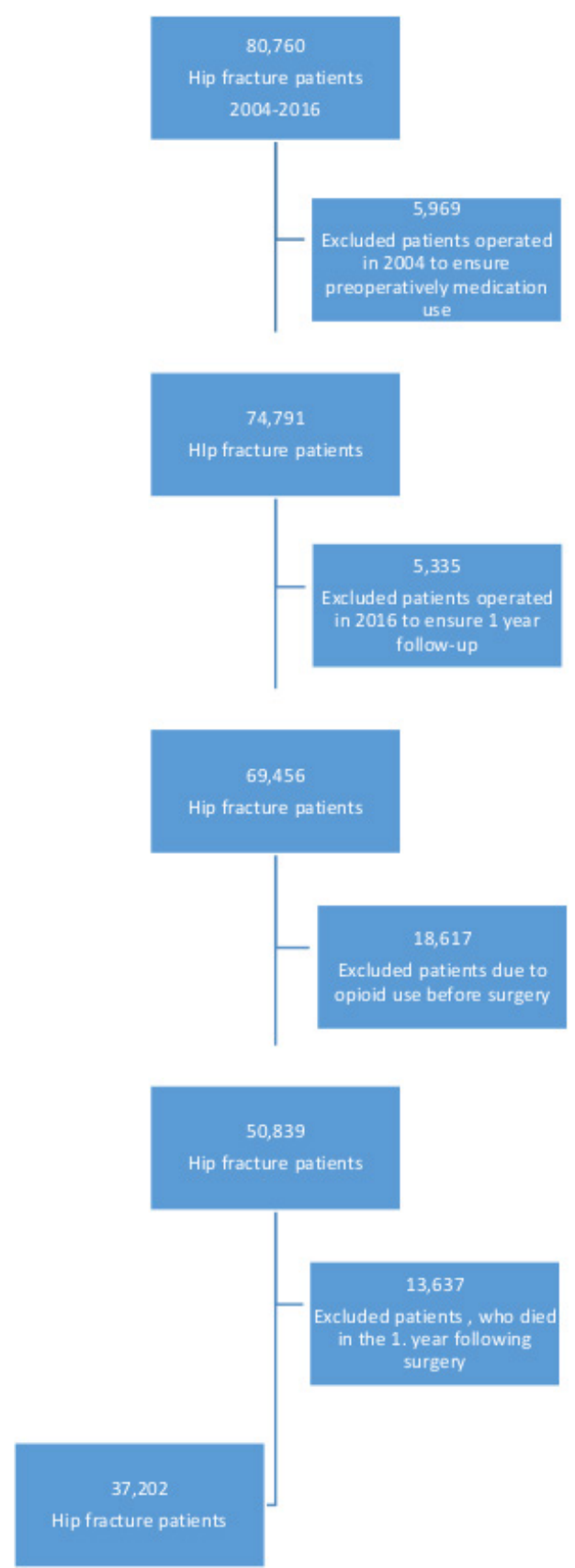

Figure 1 Flowchart of the patients from the Danish Multidisciplinary Hip Fracture Registry to the study population.

factors were considered and examined as potential risk factors for new chronic opioid use. ${ }^{89}$ From the DMHFR, we obtained information on age (in categories 65-74, $75-84$ and $\geq 85$ years), sex, fracture type (femoral neck and pertrochanteric/subtrochanteric fracture) and surgery type (osteosynthesis and total/partial hip replacement). Body Mass Index (BMI) was calculated using information on height and weight (weight in kilogram divided by height in metre squared) and divided into groups (underweight defined as BMI $<18.5$, normal as BMI 18.624.9, overweight as BMI 25-29.9 and obese as BMI $\geq 30$ ). We examined several specific medical comorbidities, including myocardial infarction, congestive heart failure, peripheral vascular disease, cerebrovascular disease, diabetes, liver disease, peptic ulcer disease, connective tissue disease, dementia, hemiplegia, chronic obstructive pulmonary disease, renal disease and cancer (table 1 ). BMI and various comorbidities were in previous studies found to be associated with increased risk of mortality and could be associated with increased risk of chronic opioid use..$^{819-21}$

We also included data on preoperative dispensing of the following comedications: non-steroidal anti-inflammatory drug (NSAID), selective serotonin reuptake inhibitors (SSRIs), any antidepressants, antipsychotics, oral corticosteroids, statins, antibiotics, hormone replacement therapy, antiosteoporosis medication, vitamin K, any anticoagulants, novel oral anticoagulant, antiplatelet drugs and heparins (online supplemental appendix 2). These drugs are included as potential risk factors because they can influence general healthcare use and behaviour, or are associated with increased mortality. ${ }^{22}$ The preoperative medication for each drug was defined as at least one dispensing in the 1 year before surgery.

\section{Statistical analyses}

For the presentation of demographic data, descriptive statistics were used and presented for the entire study population at the time of surgery and separately for patients with new chronic use. ORs with 95\% CI were calculated using multiple logistic regression and were adjusted for age and sex (adjusted OR (aOR)). Several sensitivity analyses were performed: (1) an analysis when using logistic regression and adjusting for multiple relevant factors; (2) an analysis where all patients who died within the first year were included; (3) landmark analysis at 6 months (only excluding the patients who died in the first and second quarter) and at 9 months (only excluding the patients who died in the first, second and third quarters) ${ }^{23} 24$; (4) an analysis when only including patients, who sustained a fracture of the femoral neck to analyse the treatment with total/partial hip replacement and osteosynthesis. All statistical analyses were performed in STATA V.15.

This paper was reported following the Strengthening the Reporting of Observational Studies in Epidemiology statement ${ }^{25}$ and the Reporting of studies Conducted using Observational Routinely-collected Data statement. ${ }^{26}$

\section{Patient and public involvement}

No patient was involved.

\section{RESULTS}

\section{Description of the study population}

In total, 69456 patients with hip fracture surgery were identified (figure 1). We excluded 18617 patients with hip fracture due to opioid use before surgery, leaving us with 50839 eligible patients with hip fracture. Of these, 13637 patients died within the first year. The final study population included 37202 patients with hip fracture. 


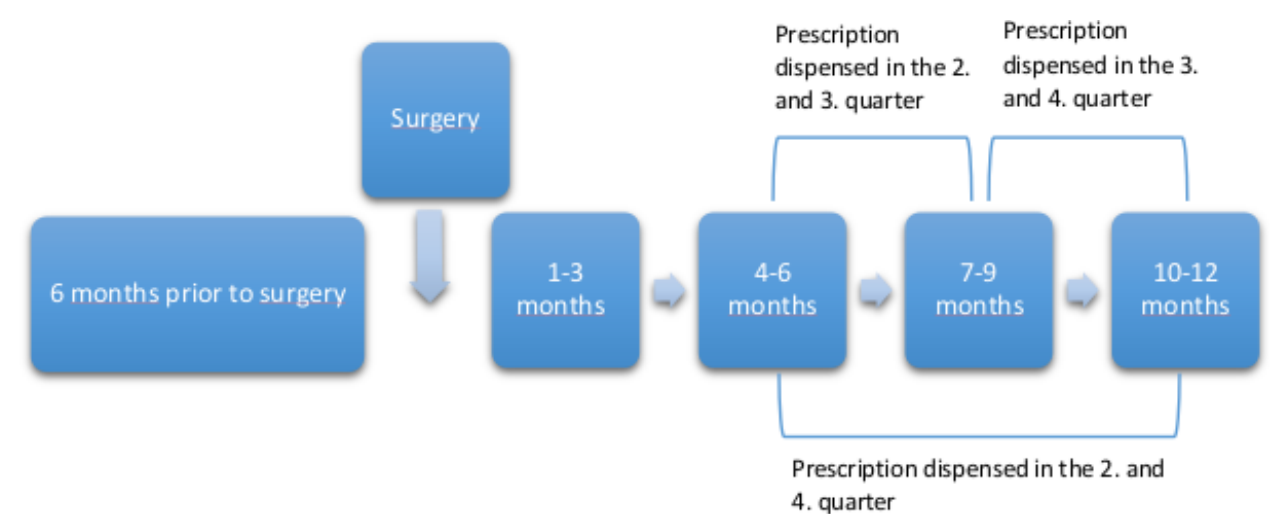

Figure 2 New chronic opioid use was defined as patients with at least two prescriptions dispensed in two of the three latter quarters in the first year following surgery.

Overall, 27133 patients $(73 \%)$ were female and the mean age at the time of surgery was 81 years (range 65-107). In our study population, 5497 (15\%) developed new chronic opioid use within 1 year of surgery.

The proportion of patients who developed new chronic opioid use in relation to all patients with hip fracture was $15 \%$ for both women and men, $13 \%$ for patients with femoral neck fractures and $16 \%$ for patients with a pertrochanteric or subtrochanteric fracture (table 1).

The characteristics of patients with new chronic opioid use were similar to the characteristics of the total population of patients with hip fracture with small differences seen in the distribution of BMI, fracture type, surgery type and preoperative medication use (table 1).

\section{Risk factors for new chronic opioid use}

The risk factors for new chronic opioid use were being underweight (aOR 1.22, 95\% CI 1.09 to 1.36), overweight (aOR $1.12,95 \%$ CI 1.04 to 1.21 ) or obese (aOR 1.57, $95 \%$ CI 1.40 to 1.76 ) with normal weight as reference and sustaining a pertrochanteric/subtrochanteric fracture (aOR $1.27,95 \%$ CI 1.20 to 1.34 ) with fracture of the femoral neck as reference. Treatment with total/partial hip replacement was associated with lower risk of new chronic opioid use, with osteosynthesis as reference (aOR $0.65,95 \%$ CI 0.60 to 0.69 ) (figure 3 and online supplemental appendix figure 1).

Preoperative use of NSAIDs (aOR 1.68, 95\% CI 1.55 to 1.83 ), SSRIs (aOR 1.42. 95\% CI 1.32 to 1.53 ), antidepressants (aOR 1.36, 95\% CI 1.24 to 1.49 ), antipsychotics (aOR 1.21, 95\% CI 1.07 to 1.35 ), corticosteroids (aOR $1.54,95 \%$ CI 1.35 to 1.76 ), statins (aOR 1.09, 95\% CI 1.02 to 1.18 ), antibiotics (aOR $1.32,95 \%$ CI 1.22 to 1.42 ), antiosteoporosis drugs (aOR 1.33, 95\% CI 1.19 to 1.49 ), anticoagulants (aOR 1.24, 95\% CI 1.17 to 1.32 ) and antiplatelet drugs (aOR 1.24, 95\% CI 1.16 to 1.32 ) were identified as risk factors for new chronic opioid use (figure 3 and online supplemental appendix figure 1 ).

The presence of the following preoperative comorbidities were further associated with risk of new chronic opioid use: cardiovascular comorbidity, diabetes, gastrointestinal diseases, dementia, COPD and renal diseases (figure 3 and online supplemental appendix figure 1).

Several sensitivity analyses were performed. The estimates from the sensitivity analysis including patients who were deceased within the first year of surgery differed slightly from the primary analysis; for example, the OR for new chronic opioid use among the youngest patients was 1.07 (95\% CI 0.99 to 1.15$)$ in the primary analysis changing to 1.45 (95\% CI 1.35 to 1.56 ) in this sensitivity analysis (please see figure 4). Results of the landmark analyses were similar to the results presented in the primary analyses, where we excluded all patients who died within 12 months of surgery (please see online supplemental appendices 2 and 3). Likewise, analyses adjusting for multiple relevant factors and those based on patients with only a fracture of the femoral neck showed results similar to those of the primary analysis (data not shown).

\section{DISCUSSION}

To our knowledge, this is the first study to examine risk factors for new chronic opioid use following hip fracture surgery among patients who were alive within the first year following surgery. In this large nationwide cohort study of 37202 patients who underwent hip fracture surgery, $15 \%$ of the patients had become new chronic opioid users within the first 12 months after surgery, of which patients with a femoral neck represented $55 \%$. We identified several patient characteristics, comorbidities and preoperative medications as possible risk factors that could be associated with new chronic opioid use after surgery.

\section{Strength and limitations}

The strength of this study is that it is a nationwide population-based cohort study with prospective, validated data and complete follow-up. In addition, we had comprehensive information on medication use and comorbidities prior to surgery, detailed clinical data on patients with hip fracture with regard to information on fracture type, surgery type and BMI and 
Table 1 Patient characteristics for the all patients with hip fracture and new chronic users

\begin{tabular}{|c|c|c|c|}
\hline & All $(n=37202)$ & New chronic user $(n=5497)$ & Proportions of \\
\hline & n (\%) & n (\%) & user (\%) \\
\hline Age (years) & & & \\
\hline Mean (SD) & $81.4(7.9)$ & $81.3(7.9)$ & \\
\hline $65-74$ & $8554(23)$ & $1302(24)$ & -15 \\
\hline $75-84$ & $15302(41)$ & $2268(41)$ & -15 \\
\hline 85 & $13346(36)$ & $1927(35)$ & -14 \\
\hline Sex & & & \\
\hline Female & $27133(73)$ & $4021(73)$ & -15 \\
\hline Male & $10069(27)$ & $1476(27)$ & -15 \\
\hline BMI group & & & \\
\hline Underweight (<18.5) & $2556(7)$ & $409(7)$ & -16 \\
\hline Normal (18.6-24.9) & $17129(46)$ & $2306(42)$ & -13 \\
\hline Overweight (25.0-29.9) & $6783(18)$ & $1046(19)$ & -15 \\
\hline Obese $(+30)$ & $8684(23)$ & $1456(26)$ & -17 \\
\hline Missing & $6853(18)$ & $1083(20)$ & -16 \\
\hline Fracture type & & & \\
\hline Femoral neck & $20288(55)$ & $2724(50)$ & -13 \\
\hline Pertrochanteric/subtrochanteric & $16914(45)$ & $2773(50)$ & -16 \\
\hline Surgery type & & & \\
\hline Osteosynthesis & $25489(69)$ & $4179(76)$ & -16 \\
\hline Total/partial hip replacement & $11713(31)$ & $1318(24)$ & -11 \\
\hline Cardiovascular comorbidities & & & \\
\hline Myocardial infarction & $1629(4)$ & $274(5)$ & -17 \\
\hline Congestive heart failure & $2340(6)$ & $444(8)$ & -19 \\
\hline Peripheral vascular disease & $2153(6)$ & $429(8)$ & -20 \\
\hline Cerebrovascular disease & $6143(17)$ & $1035(19)$ & -17 \\
\hline Diabetes & $3055(8)$ & $573(10)$ & -19 \\
\hline Gastrointestinal comorbidities & & & \\
\hline Liver disease & $325(1)$ & $88(2)$ & -27 \\
\hline Peptic ulcer disease & $1526(4)$ & $335(6)$ & -22 \\
\hline Connective tissue disease & $1387(4)$ & $229(4)$ & -17 \\
\hline Neurological comorbidities & & & \\
\hline Dementia & $2592(7)$ & $434(8)$ & -17 \\
\hline Hemiplegia & $89(0)$ & $16(0)$ & -18 \\
\hline COPD & $3365(9)$ & $643(12)$ & -19 \\
\hline Renal disease & $828(2)$ & $144(3)$ & -17 \\
\hline Any cancer & $4623(12)$ & $719(13)$ & -16 \\
\hline Preoperative medication & & & \\
\hline NSAID & $3904(10)$ & $817(15)$ & -21 \\
\hline SSRI & $5959(16)$ & $1103(20)$ & -19 \\
\hline Corticosteroid & $1427(4)$ & $295(5)$ & -21 \\
\hline Anticoagulants & $13062(35)$ & 2134 (39) & -16 \\
\hline Statins & $6949(19)$ & $1088(20)$ & -16 \\
\hline Antibiotics & 6479 (17) & $1106(20)$ & -17 \\
\hline
\end{tabular}




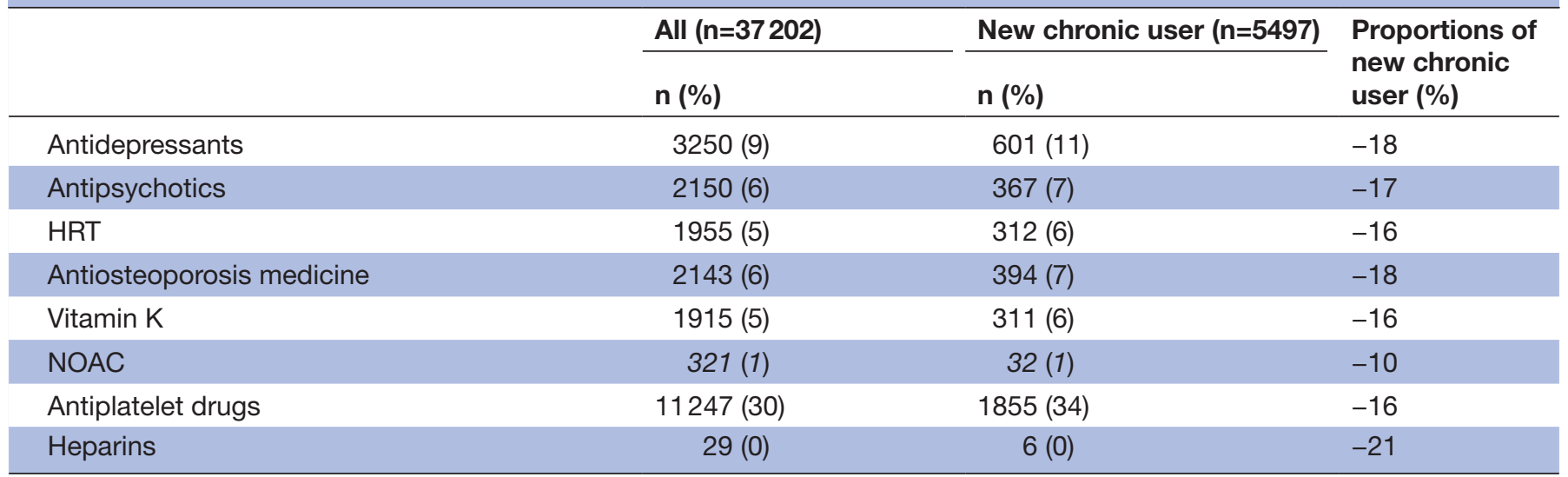

ORs with $95 \%$ Cls.

BMI, Body Mass Index; COPD, chronic obstructive pulmonary disease; HRT, hormone replacement Therapy; NOAC, novel oral anticoagulant; NSAID, non-steroidal anti-inflammatory drug; SSRI, selective serotonin reuptake inhibitor.

opioid information based on dispensing data rather than patient-reported data.

Our study also has some limitations. First, there is no consensus on how to define chronic opioid use. Previous studies have defined chronic opioid use as postoperative opioid prescription fulfilment between 90 and 180 days, ${ }^{27}$ or opioid prescriptions for 120 non-consecutive days. ${ }^{8}$ The heterogeneity in definitions for chronic opioid use limits the ability to compare our results with previous studies. Moreover, dispensing data provides an imperfect representation of true preoperative medication use, and we were unable to ascertain the intended indications of opioid prescriptions. We know that patients have collected the opioid prescription at the pharmacy, but we have no knowledge regarding the patient's compliance. Even so, using prescription opioid dispensing data is considered a better measure of medication use than most alternative measures. ${ }^{28}$ Second, we excluded all deceased patients within the first year following surgery, which might have compromised the external validity of our study. Thus, we can only conclude that identified risk factors for new chronic opioid use apply for patients with hip fracture that survived 1 year after surgery. The number of deceased patients was substantial. A total of 13637 of the 50839 patients with hip fracture died within the first year (please see online supplemental appendix figure 4). Including these in the study population would have an impact on our results (please see figure 4). A total of 751 of the 13637 deceased patients with hip fracture were potentially eligible to be included in our study as new chronic opioid users. These patients had redeemed two opioid prescriptions in either the second, third or fourth quarter cf. our definition (please see online supplemental appendix figure 4). In general, immortal time can bias the effect estimates in pharmacoepidemiological studies. ${ }^{23}$ The landmark approach is one of the methods often used when addressing immortal time bias; however, its simplicity comes at the cost of difficulty in interpreting the results. ${ }^{23} 24$ The performed landmark analyses illustrated that including the deceased patients from the third and fourth quarters in our primary analysis would not affect our results substantially (please see online supplemental appendixs 2 and 3). The effects of the different analytical assumptions on the results are summarised in figure 5, showing the changes from analysis to analysis plotted side by side. Third, we were not able to include reoperation in our analysis. This may have overestimated the risk of new chronic opioid use in younger female patients, since patients with hip fracture are at risk of reoperation, which may lead to prolonged or restarted opioid use. We know that $6 \%$ of patients with hip fracture are reoperated ${ }^{29}$ and that individuals aged 80 years or younger and male gender are associated with risk of reoperation. ${ }^{30}$

\section{Comparison with previous studies}

Only two studies have reported prescription opioid use after hip fracture surgery. Simoni et al found that 28\% of Danish patients with hip fracture had dispensed an opioid prescription within the first year after surgery. Moreover, $17 \%$ of the patients who were opioid non-users before surgery had dispensed an opioid prescription 1 year after surgery. ${ }^{4}$ That study, however, did not examine chronic opioid use, only opioid use in general defined as one dispensed opioid prescription. In a similar study, Lindestrand et al conducted a medical record review from a single institutional with 416 patients and found $2.9 \%$ of previous opioid nonuser patients were opioid users at 6 months. The study reported further that osteoporosis and opioid use prior to admission were predictors for postoperative opioid use at 6 months. In contrast to our study, they did not define opioid use, and the follow-up period ended at 6 months after hip fracture. ${ }^{5}$ We studied the risk factors in a large nationwide setup, whereby we 


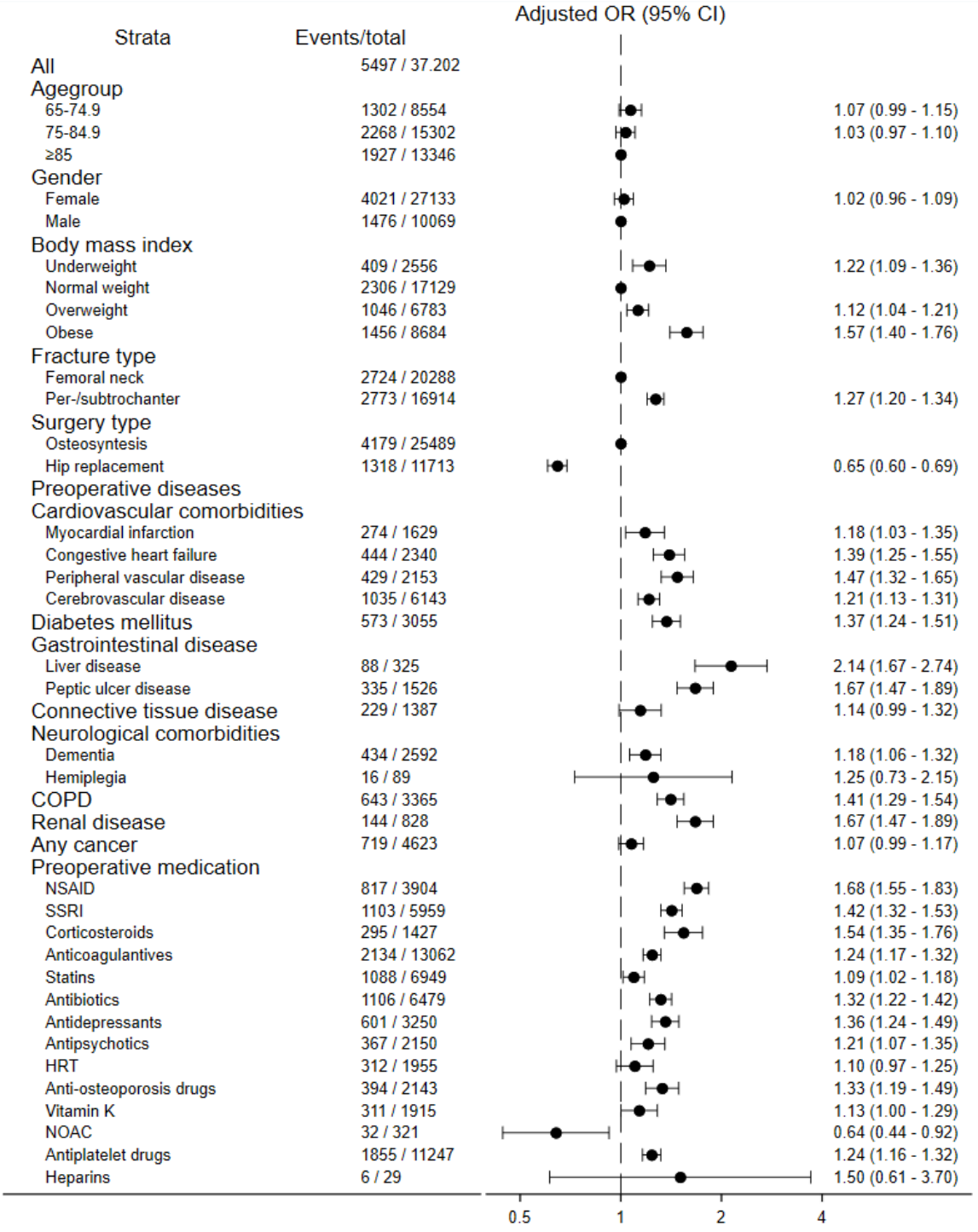

Figure 3: Risk factors for developing new chronic opioid use after hip fracture surgery among patient with no opioid use before surgery in those who were alive 12 months after surgery, odds ratios adjusted for age and sex.

COPD: Chronic pulmonary disease, NSAID: nonsteroidal anti-inflammatory drug, SSRI: selective serotonin reuptake inhibitors, HRT: Hormone Replacement Therapy, NOAC: novel oral anticoagulant. Odds ratios (ORs) with $95 \%$ confidence intervals (CI)

Figure 3 Risk factors for developing new chronic opioid use after hip fracture surgery among patients with no opioid use before surgery in those who were alive 12 months after surgery, ORs adjusted for age and sex. ORs with 95\% Cls. COPD, chronic obstructive pulmonary disease; HRT, hormone replacement therapy; NOAC, novel oral anticoagulant; NSAID, nonsteroidal anti-inflammatory drug; SSRI, selective serotonin reuptake inhibitor.

uncovered trends across the entire country and not only from a single institution.

In general, there is evidence that younger biological age is a predictor of persistent opioid use in the general surgical population. ${ }^{68}$ This is explained by a wide variety of factors in the ageing population such as a decline in the production of several proteins and neuropeptides, a decline of the immune response and an increase in the inflammatory response. ${ }^{31}$ Our study shows the same tendency.

Several studies have shown the prevalence of chronic pain and consumption of opioids tend to be higher in women than in men. ${ }^{17} 32$ Psychological, biological, cultural and social factors all play a role in the differences between the sex in pain responses and management. ${ }^{1733}$ Our study demonstrates a weak association between the female sex and new chronic opioid use after hip fracture surgery.

Overweight and obesity have been shown to be associated with a proinflammatory state after surgery inducing hyperalgesia, suggesting an increase in opioid use, which correlates with findings by Westermann et al of an association between obesity and prolonged postoperative opioid use. ${ }^{19} 2034$ This is in 


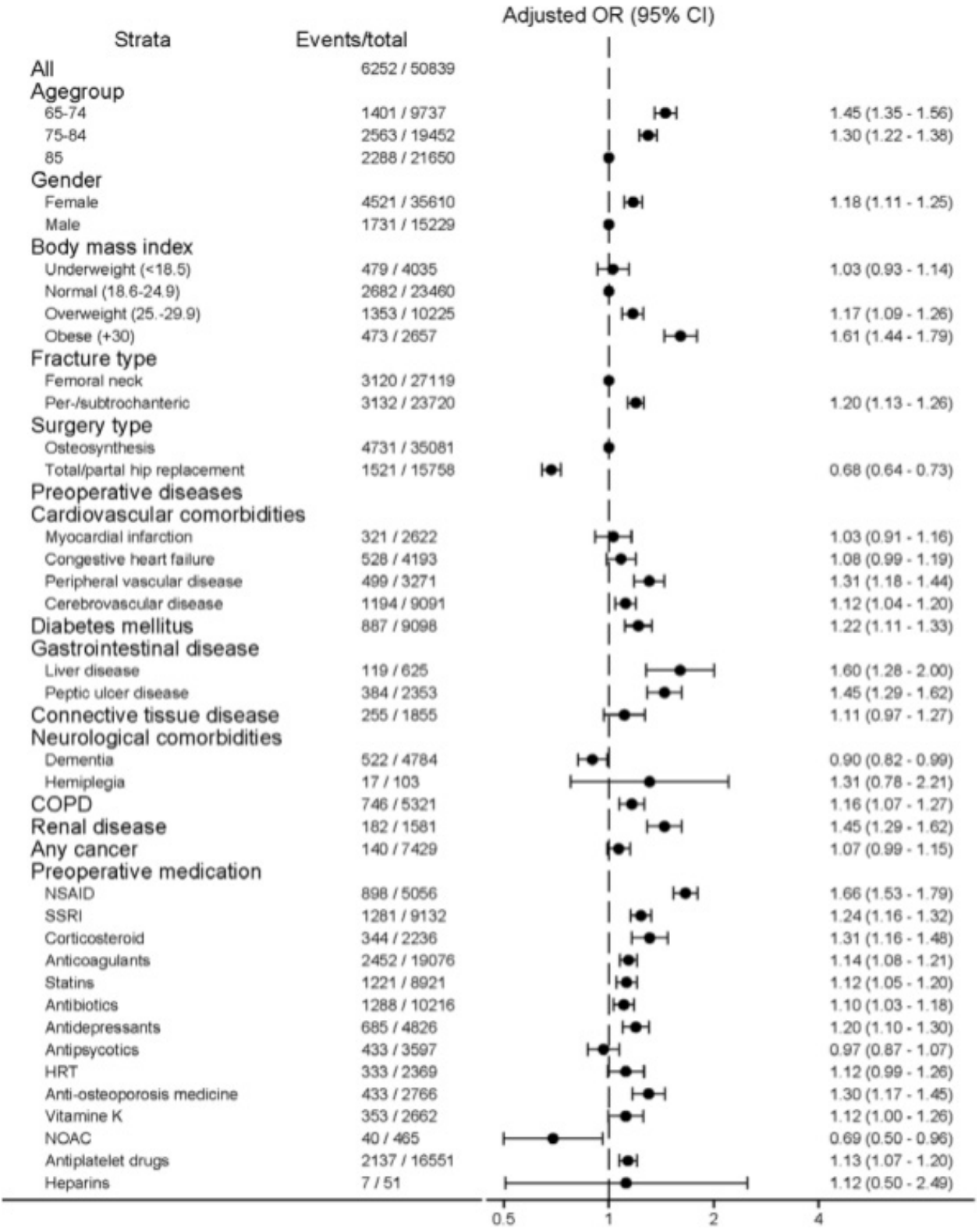

Figure 4 Risk factors for developing new chronic opioid use after hip fracture surgery among patients with no opioid use before surgery, including patients who were alive and those who died within 12 months of surgery, ORs adjusted for age and sex. ORs with 95\% Cls. COPD, chronic obstructive pulmonary disease; HRT, hormone replacement therapy; NOAC, novel oral anticoagulant; NSAID, non-steroidal anti-inflammatory drug; SSRI, selective serotonin reuptake inhibitor.

line with our findings of an association between overweight and obesity and developing a new chronic opioid use after surgery.

Our data suggest that fracture type and surgery type are associated with new chronic opioid use. Patients with hip fracture with a trochanteric fracture experience more and severe pain than patients with femoral neck fractures. ${ }^{35}$ Similarly, patients with osteosynthesis experience more pain than the patients with a stable arthroplasty. ${ }^{36}$ The reported mechanisms are shortening of the limb length and range of motion limitations. ${ }^{36}$ Another explanation to why surgery type is associated with new chronic opioid use could be that these patients might have a higher rate of reoperation converting to a total hip arthroplasty performed by a more experienced surgeon. However, we do not have data to support this statement.

Several preoperative comorbidities were associated with risk of new chronic opioid use after surgery. Although we excluded all patients with hip fracture with prior use of opioids, it is possible that some patients had an unmanaged pain condition prior to surgery. These patients may have continued to use prescription opioids intended for treating postsurgical pain in order to treat their pre-existing chronic pain. ${ }^{6}$ Inacio $e t$ al support this behaviour as they found back pain prior to surgery was associated with chronic opioid use. ${ }^{8}$ Comorbidities associated with unrelieved chronic pain conditions are heart failure and COPD. These comorbidities have been associated with chronic 
A.

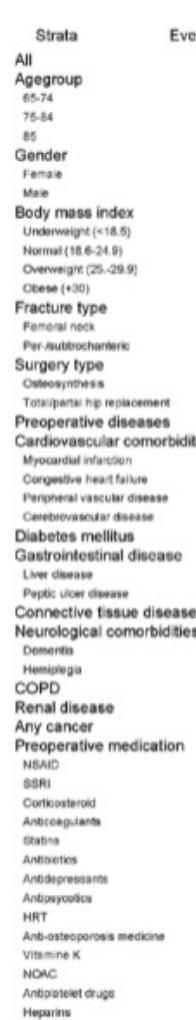

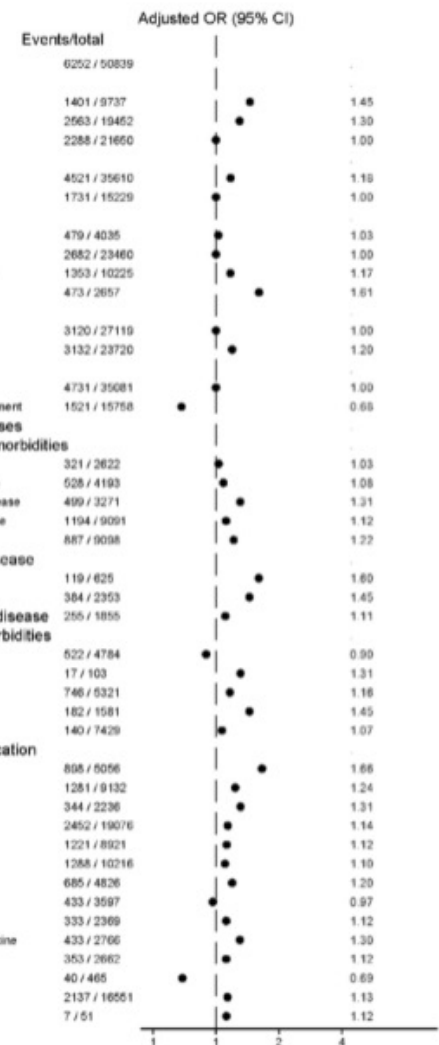

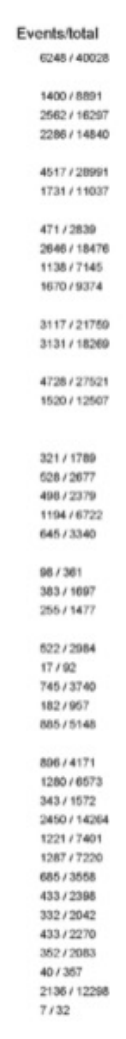

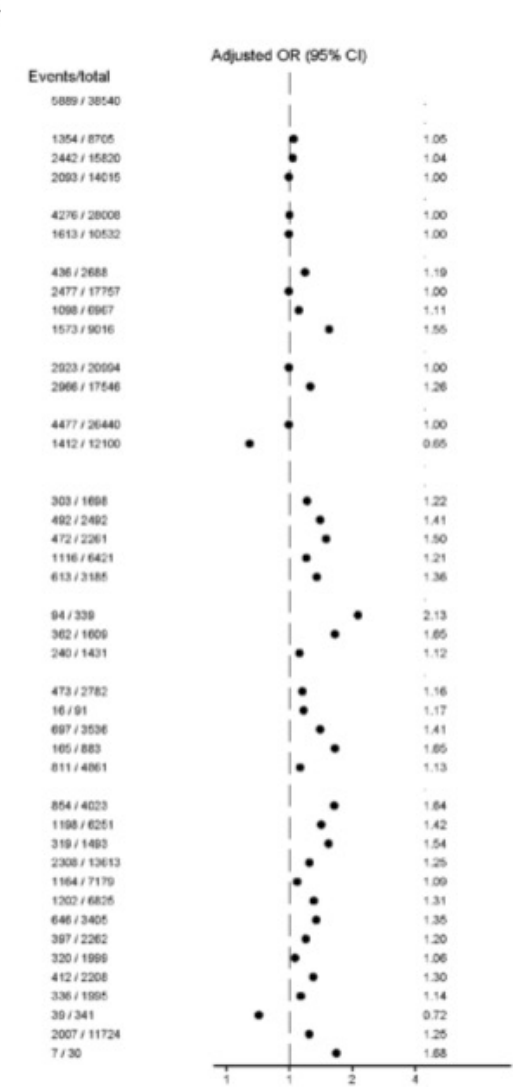

Figure 5 United forest plot. (A) Analysis, when including patients who were alive and those who died within 12 months of surgery. (B) Landmark analysis at 6 months: analysis, when including patients with no opioid use before surgery and excluding the patients who died in the first and second quarters of surgery. (C) Landmark analysis at 9 months: analysis, when including patients with no opioid use before surgery and excluding the patients who died in the first, second and third quarters of surgery. ORs adjusted for age and sex. COPD, chronic obstructive pulmonary disease; HRT, hormone replacement therapy; NOAC, novel oral anticoagulant; NSAID, non-steroidal anti-inflammatory drug; SSRI, selective serotonin reuptake inhibitor.

opioid use, which concords with our study. ${ }^{6}$ Diabetes has also been associated with a constant chronic inflammatory state inducing neuropathy, which has also been associated with unrelieved chronic pain. This mechanism is a potential risk factor for chronic pain, which is in accordance with our study. ${ }^{6} 8$ Other comorbidities have also been associated with chronic pain and chronic opioid use such as liver disease and depression. ${ }^{8}$ By knowing the impact of these comorbidities on the risk of new chronic opioid use, attainment of a greater focus on comorbidity preoperative and postoperative may reduce new chronic opioid use after surgery.

Medication use is frequent in patients with hip fracture, and nearly all of the included medications in our study were identified as a risk factor for chronic opioid use. ${ }^{37}$ Medication use is closely related to comorbidities. Treatment of chronic medical conditions is a complex task that require multidisciplinary approach. It is possible that surgeons and patients are preoccupied with attempting to manage chronic pain conditions leaving long-term opioid use as a secondary priority. Some drugs, when taken on their own or in combination, might change the level of sensitivity to opioids, which could result in patients who continue to take opioids even though their level of pain decreases over time and does not necessarily coincide with the prescribed opioid dose.

\section{Chronic opioid use}

There are no standard guidelines in Denmark for postdischarge clinical follow-up of patients with hip fracture. However, many orthopaedic and geriatric departments focus on the reduction of prescription opioid exposure after surgery by instituting a plan for opioid tapering. Patients do not receive follow-up appointments in outpatient clinics or at the general practitioner unless they take the initiative themselves. Since patients with hip fracture often are characterised as being frail, receiving several medications and having multimorbidity, they may lack the resources to follow such a tapering plan. Thus, it is important to ensure that patients are well informed and included in the development of a tapering plan, and to understand the risks and benefits of prescription opioids for the treatment of postsurgical pain. However, it is important to note that not all hip fracture surgeries are successful, and some patients may experience a 
greater level of postsurgical pain and postsurgical pain treatment.

\section{CONCLUSION}

In this large nationwide cohort study, $15 \%$ of the patients who underwent hip fracture surgery developed new chronic opioid use. We identified underweight and overweight, obesity, pertrochanteric or subtrochanteric fracture, preoperative use of several medications and presence of several comorbidities as risk factors associated with the risk of new chronic opioid use after hip fracture surgery.

By identifying risk factors, we can reduce the number of new chronic opioid users by developing more effective preventive intervention strategies targeted to the patients with the identified risk factors. In addition, the identified risk factors are also relevant for clinicians in order to advise patients appropriately before surgery about their risk of chronic postsurgical opioid use.

\section{Author affiliations}

${ }^{1}$ Department of Clinical Epidemiology, Aarhus University Hospital, Aarhus N, Denmark

${ }^{2}$ Department of Orthopaedic Surgery, Lillebaelt Hospital, Vejle, Denmark

${ }^{3}$ Department of Regional Health Research, University of Southern Denmark Faculty of Health Sciences, Odense, Denmark

${ }^{4}$ Department of Orthopaedic Surgery and Traumatology, Odense University Hospital, Odense, Denmark

${ }^{5}$ Department of Clinical Research, University of Southern Denmark, Odense,

Denmark

${ }^{6}$ Department of Anesthesiology and Intensive Care, Aarhus University Hospital, Aarhus, Denmark

\section{Twitter Nina McKinnon Edwards @NinaMcKEdwards}

Acknowledgements We thank the staff of the hospital departments caring for the patients with hip fracture for their continuous effort and contribution to acquisition of the data in the Danish Multidisciplinary Hip Fracture Registry.

Contributors NMcKE, CV, SO, LN, CFC and ABP contributed to the conception or design of the study and interpretation of data, and drafted the manuscript or revised it critically. NMcKE carried out the analytical aspects of the study. All authors gave their final approval and agreement to be accountable for all aspects of the work.

Funding The authors have not declared a specific grant for this research from any funding agency in the public, commercial or not-for-profit sectors.

Competing interests None declared.

Patient consent for publication Not required.

Ethics approval The study was approved by the Danish Data Protection Agency's journal number (2015-57-0002) and Aarhus University's journal number (2016051-000001), record number 880 .

Provenance and peer review Not commissioned; externally peer reviewed.

Data availability statement № data are available. No additional data are available.

Supplemental material This content has been supplied by the author(s). It has not been vetted by BMJ Publishing Group Limited (BMJ) and may not have been peer-reviewed. Any opinions or recommendations discussed are solely those of the author(s) and are not endorsed by BMJ. BMJ disclaims all liability and responsibility arising from any reliance placed on the content. Where the content includes any translated material, BMJ does not warrant the accuracy and reliability of the translations (including but not limited to local regulations, clinical guidelines, terminology, drug names and drug dosages), and is not responsible for any error and/or omissions arising from translation and adaptation or otherwise.
Open access This is an open access article distributed in accordance with the Creative Commons Attribution Non Commercial (CC BY-NC 4.0) license, which permits others to distribute, remix, adapt, build upon this work non-commercially, and license their derivative works on different terms, provided the original work is properly cited, appropriate credit is given, any changes made indicated, and the use is non-commercial. See: http://creativecommons.org/licenses/by-nc/4.0/.

\section{ORCID iDs}

Nina McKinnon Edwards http://orcid.org/0000-0002-0473-228X

Claus Varnum http://orcid.org/0000-0002-0625-5691

Søren Overgaard http://orcid.org/0000-0001-6829-4787

Lone Nikolajsen http://orcid.org/0000-0002-2705-7088

Christian Fynbo Christiansen http://orcid.org/0000-0002-0727-953X

Alma Becic Pedersen http://orcid.org/0000-0002-3288-9401

\section{REFERENCES}

1 Cooper C, Cole ZA, Holroyd CR, et al. Secular trends in the incidence of hip and other osteoporotic fractures. Osteoporos Int 2011;22:1277-88.

2 Kristiansen NS, Kristensen PK, Nørgård BM, et al. Off-Hours admission and quality of hip fracture care: a nationwide cohort study of performance measures and 30-day mortality. Int J Qual Health Care 2016;28:324-31.

3 Summers S, Grau L, Massel D, et al. Opioid use disorders are associated with perioperative morbidity and mortality in the hip fracture population. J Orthop Trauma 2018;32:238-44.

4 Simoni AH, Nikolajsen L, Olesen AE, et al. Opioid use after hip fracture surgery: a Danish nationwide cohort study from 2005 to 2015. Eur J Pain 2019;23:1309-1317.

5 Lindestrand AG, Christiansen MLS, Jantzen C, et al. Opioids in hip fracture patients: an analysis of mortality and post Hospital opioid use. Injury 2015;46:1341-5.

6 Clarke H, Soneji N, Ko DT, et al. Rates and risk factors for prolonged opioid use after major surgery: population based cohort study. BMJ 2014;348:g1251.

7 Teng Z, Zhu Y, Wu F, et al. Opioids contribute to fracture risk: a metaanalysis of 8 cohort studies. PLoS One 2015;10:e0128232.

8 Inacio MCS, Hansen C, Pratt NL, et al. Risk factors for persistent and new chronic opioid use in patients undergoing total hip arthroplasty: a retrospective cohort study. BMJ Open 2016;6:e010664.

9 Bedard NA, Pugely AJ, Dowdle SB, et al. Opioid use following total hip arthroplasty: trends and risk factors for prolonged use. $J$ Arthroplasty 2017;32:3675-9.

10 Schmidt M, Schmidt SAJ, Adelborg K, et al. The Danish health care system and epidemiological research: from health care contacts to database records. Clin Epidemiol 2019;11:563-91.

11 Schmidt M, Pedersen L, Sørensen HT. The Danish civil registration system as a tool in epidemiology. Eur J Epidemiol 2014;29:541-9.

12 Kristensen PK, Röck ND, Christensen HC, et al. The Danish multidisciplinary hip fracture registry 13-year results from a population-based cohort of hip fracture patients. Clin Epidemiol 2020;12:9-21.

13 Hjelholt TJ, Edwards NM, Vesterager JD, et al. The positive predictive value of hip fracture diagnoses and surgical procedure codes in the Danish multidisciplinary hip fracture registry and the Danish national patient registry. Clin Epidemiol 2020;12:123-31.

14 Johannesdottir SA, Horváth-Puhó E, Ehrenstein V, et al. Existing data sources for clinical epidemiology: the Danish national database of Reimbursed prescriptions. Clin Epidemiol 2012;4:303-13.

15 Schmidt M, Schmidt SAJ, Sandegaard JL, et al. The Danish national patient registry: a review of content, data quality, and research potential. Clin Epidemiol 2015;7:449-90.

16 Thygesen SK, Christiansen CF, Christensen S, et al. The predictive value of ICD-10 diagnostic coding used to assess Charlson comorbidity index conditions in the population-based Danish national Registry of patients. BMC Med Res Methodol 2011;11:83.

17 Birke H, Ekholm O, Sjøgren P, et al. Long-Term opioid therapy in Denmark: a disappointing journey. Eur J Pain 2017;21:1516-27.

18 Treede R-D, Rief W, Barke A, et al. A classification of chronic pain for ICD-11. Pain 2015;156:1003-7.

19 Westermann RW, Mather RC. 3Rd, Bedard Na, et al. prescription opioid use before and after hip arthroscopy: a caution to prescribers. Arthroscopy : the journal of arthroscopic \& related surgery : official publication of the Arthroscopy Association of North America and the International Arthroscopy Association 2019.

20 Anciano Granadillo V, Cancienne JM, Gwathmey FW, et al. Perioperative opioid analgesics and hip arthroscopy: trends, 
risk factors for prolonged use, and complications. Arthroscopy 2018;34:2359-67.

21 Skrejborg P, Petersen KK, Kold S, et al. Presurgical comorbidities as risk factors for chronic postsurgical pain following total knee replacement. Clin J Pain 2019;35:577-82.

22 Bruun SB, Petersen I, Kristensen NR, et al. Selective serotonin reuptake inhibitor use in hip fracture patients: a Danish nationwide prevalence study. Acta Orthop 2019;90:33-9.

23 Gleiss A, Oberbauer R, Heinze G. An unjustified benefit: immortal time bias in the analysis of time-dependent events. Transp/ Int 2018;31:125-30.

$24 \mathrm{Mi} \mathrm{X}$, Hammill BG, Curtis LH, et al. Use of the landmark method to address immortal person-time bias in comparative effectiveness research: a simulation study. Stat Med 2016;35:4824-36.

25 von Elm E, Altman DG, Egger M, et al. The strengthening the reporting of observational studies in epidemiology (STROBE) statement: guidelines for reporting observational studies. J Clin Epidemiol 2008;61:344-9.

26 Benchimol El, Smeeth L, Guttmann A, et al. The reporting of studies conducted using observational Routinely-collected health data (record) statement. PLoS Med 2015;12:e1001885.

27 Brummett CM, Waljee JF, Goesling J, et al. New persistent opioid use after minor and major surgical procedures in US adults. JAMA Surg 2017;152:e170504.

28 Schneeweiss S, Avorn J. A review of uses of health care utilization databases for epidemiologic research on therapeutics. J Clin Epidemiol 2005;58:323-37.
29 Gjertsen J-E, Dybvik E, Furnes O, et al. Improved outcome after hip fracture surgery in Norway. Acta Orthop 2017;88:505-11.

30 Moerman S, Mathijssen NMC, Tuinebreijer WE, et al. Hemiarthroplasty and total hip arthroplasty in 30,830 patients with hip fractures: data from the Dutch arthroplasty register on revision and risk factors for revision. Acta Orthop 2018;89:509-14.

31 Pickering G, Jourdan D, Millecamps M, et al. Age-Related impact of neuropathic pain on animal behaviour. Eur $J$ Pain 2006;10:749-55.

32 Campbell $\mathrm{Cl}$, Weisner $\mathrm{C}$, Leresche L, et al. Age and gender trends in long-term opioid analgesic use for noncancer pain. Am J Public Health 2010;100:2541-7.

33 Riley JL, Robinson ME, Wise EA, et al. Sex differences in the perception of noxious experimental stimuli: a meta-analysis. Pain 1998;74:181-7.

34 Sommer C, Kress M. Recent findings on how proinflammatory cytokines cause pain: peripheral mechanisms in inflammatory and neuropathic hyperalgesia. Neurosci Lett 2004;361:184-7.

35 Kristensen MT. Hip fracture-related pain strongly influences functional performance of patients with an intertrochanteric fracture upon discharge from the hospital. Pm R 2013;5:135-41.

36 Salpakoski A, Kallinen M, Kiviranta I, et al. Type of surgery is associated with pain and walking difficulties among older people with previous hip fracture. Geriatr Gerontol Int 2016;16:754-61.

37 Komagamine J, Hagane K. Intervention to improve the appropriate use of polypharmacy for older patients with hip fractures: an observational study. BMC Geriatr 2017; 17:288. 